\title{
Appearance Radii in Medial Axis Test Mask for Small Planar Chamfer Norms*
}

\author{
Jérôme Hulin and Édouard Thiel \\ Laboratoire d'Informatique Fondamentale de Marseille (LIF, UMR 6166), \\ Aix-Marseille Université, \\ 163 Avenue de Luminy, Case 901, 13288 Marseille cedex 9, France \\ \{Jerome.Hulin, Edouard.Thiel\}@lif.univ-mrs.fr
}

\begin{abstract}
The test mask $\mathcal{T}_{\mathcal{M}}$ is the minimum neighbourhood sufficient to extract the medial axis of any discrete shape, for a given chamfer distance mask $\mathcal{M}$. We propose an arithmetical framework to study $\mathcal{T}_{\mathcal{M}}$ in the case of chamfer norms. We characterize $\mathcal{T}_{\mathcal{M}}$ for $3 \times 3$ and $5 \times 5$ chamfer norm masks, and we give an algorithm to compute the appearance radius of the vector $(2,1)$ in $\mathcal{T}_{\mathcal{M}}$.
\end{abstract}

Keywords: medial axis, chamfer norm, distance transform.

\section{Introduction}

The medial axis MA of a shape $\mathcal{S}$ is a representation having numerous applications in image analysis, computer vision, and several other fields. Given a distance $d$, we consider the distance balls which are included in $\mathcal{S}$. A ball $B$ is maximal in $\mathcal{S}$ if $B$ is not included in any single other ball included in $\mathcal{S}$. The MA of $\mathcal{S}($ for $d$ ) is the set of centres and radii of all maximal balls in $\mathcal{S}[1$. Since MA is a covering, it is a reversible coding. In general, MA is not a minimal representation; we know that finding a minimum covering subset of MA is an NP-hard problem [2]. Variations on MA include for example the reduced MA [3] or the MA in higher resolution [4.

The MA can be efficiently detected by local tests on a distance transform DT. A binary image $I$ is composed of shape points and background points; the DT of $I$ is a copy of $I$, where each shape point is labelled with its distance to the background. The main discrete distances used are the chamfer (or weighted) distances [5] and the Squared Euclidean Distance (SED) [6].

The characterization of MA is simple for distances $d_{4}$ and $d_{8}$ (the simplest chamfer distances, also known as $\ell_{1}$ and $\ell_{\infty}$ norms). In [1], Pfaltz and Rosenfeld have shown that MA is the set of local maxima in DT, using the 4 - and the 8-neighbourhood, respectively. Arcelli and Sanniti di Baja 7] have studied the $\langle 3,4\rangle$ chamfer norm: after lowering some DT labels, the local maxima criterion over the 8-neighbourhood still holds. A general method, named the LUT (lookup table) method, has then been proposed by Borgefors in $[8$ for the $\langle 5,7,11\rangle$ chamfer norm, with a local test on the $5 \times 5$ neighbourhood.

\footnotetext{
^ Work supported in part by ANR grant BLAN06-1-138894 (projet OPTICOMB).
} 
In the LUT method, the LUT gives for each DT value of a point $p$ and each neighbour $q$ of $p$, the minimum DT value of $q$ that would forbid $p$ to be in MA. Naturally, the size of a sufficient neighbourhood depends on the width of the shape. The test mask $\mathcal{T}(R)$ is the minimum test neighbourhood sufficient to extract the MA of all shapes whose inner radii are no greater than $R$, the inner radius of $\mathcal{S}$ being the radius of a larger ball included in $\mathcal{S}$.

Algorithms to compute both LUT and $\mathcal{T}(R)$ are given by Rémy and Thiel in arbitrary dimension for chamfer norms and SED 910, with code available in dimensions 2 to 6 in 11. In recent papers, Normand and Évenou have proposed a faster method for chamfer norms in 2D and 3D based on a polytope representation of chamfer balls 1213 .

We now aim to understand the properties of $\mathcal{T}(R)$. We have recently shown for SED in $\mathbb{Z}^{n}$ that $\mathcal{T}(R)$ tends to the set of visible vectors when $R$ tends to infinity [14], a vector being visible if its coordinates are coprime.

In this paper we focus on 2-dimensional chamfer norms, which have been studied in 15. We examine the test masks for small chamfer norms, namely the $3 \times 3$ chamfer $\langle a, b\rangle$ and $5 \times 5$ chamfer $\langle a, b, c\rangle$ masks. We show that for these norms, the chamfer mask itself is a sufficient test neighbourhood for MA. Moreover, the neighbour $\vec{c}(2,1)$ may not be in $\mathcal{T}$ for $5 \times 5$ norms; we give an arithmetical criterion to know whether $\vec{c}$ belongs to $\mathcal{T}$. The appearance radius of a vector $\vec{v}$ is the smallest $R$ for which $\vec{v} \in \mathcal{T}(R)$. We link our problem to the Frobenius problem [16, so as to provide a simple algorithm which computes the appearance radius of $\vec{c}$ in time $\mathcal{O}(b c)$. After some preliminary definitions in Section 2, we present the test mask in Section 3 and discuss the case of $3 \times 3$ chamfer norms. Section 4 deals with $5 \times 5$ chamfer norms, while Section 5 is devoted to the appearance radius of $\vec{c}$. We finally conclude in Section 6 .

Due to the lack of space, the proofs are not in the paper; they are available in an electronic annex [17.

\section{Preliminaries}

\subsection{The Discrete Space $\mathbb{Z}^{n}$}

In the following, we consider $\mathbb{Z}^{n}$ both as a $n$-dimensional $\mathbb{Z}$-module (i.e., a discrete vector space) and as its associated affine space; we set $\mathbb{Z}_{*}^{n}=\mathbb{Z}^{n} \backslash\{0\}$. The Cartesian coordinates of a vector $\vec{v}$ are denoted by $\left(v_{1}, \ldots, v_{n}\right)$. A point $p$ is called visible if there is no lattice point between $O$ and $p$ on the line segment $[O p]$, i.e., if the coordinates of $p$ are coprime. An $n$-dimensional shape $\mathcal{S}$ is a subset of $\mathbb{Z}^{n}$.

We call $\Sigma^{n}$ the group of axial and diagonal symmetries in $\mathbb{Z}^{n}$ about centre $O$. The cardinal of the group is \# $\Sigma^{n}=2^{n} n$ ! (which is 8,48 and 384 for $n=2$, 3 and 4$)$. A shape $\mathcal{S}$ is said to be G-symmetrical if for every $\sigma \in \Sigma^{n}$ we have $\sigma(\mathcal{S})=\mathcal{S}$. The generator of a set $\mathcal{S} \subseteq \mathbb{Z}^{n}\left(\right.$ or $\left.\mathbb{R}^{n}\right)$ is $\mathrm{G}(\mathcal{S})=\left\{\left(p_{1}, \ldots, p_{n}\right) \in \mathcal{S}\right.$ : $\left.0 \leqslant p_{n} \leqslant p_{n-1} \leqslant \ldots \leqslant p_{1}\right\}$. 
Given two vectors $\vec{u}, \vec{v} \in \mathbb{Z}^{n}$ and a point $p \in \mathbb{Z}^{n}$, we define the vector cone $\mathcal{C}(\vec{u}, \vec{v})=\vec{u} \mathbb{N}+\vec{v} \mathbb{N}=\{\alpha \vec{u}+\beta \vec{v}: \alpha, \beta \in \mathbb{N}\}$. Also, we define the affine cone $\mathcal{C}(p, \vec{u}, \vec{v})=p+\mathcal{C}(\vec{u}, \vec{v})$.

\subsection{The Frobenius Problem and Representable Integers}

Let $x, a_{1}, \ldots, a_{n}$ be natural numbers, $x$ is said $\left(a_{1}, \ldots, a_{n}\right)$-representable if $x \in$ $a_{1} \mathbb{N}+\cdots+a_{n} \mathbb{N}$, i.e., if there are $n$ non-negative integers $\lambda_{1}, \ldots, \lambda_{n}$ s.t. $x=\lambda_{1} a_{1}+$ $\cdots+\lambda_{n} a_{n}$. The largest natural number that is not $\left(a_{1}, \ldots, a_{n}\right)$-representable is called the Frobenius number, denoted by $g\left(a_{1}, \ldots, a_{n}\right)$; it exists iff $a_{1}, \ldots, a_{n}$ are coprime. Sylvester proved that if $a$ and $b$ are coprime, then $g(a, b)=a b-a-b$ (see [16] for a complete study). For example, the set of all $(4,7)$-representable integers are $4 \mathbb{N}+7 \mathbb{N}=\{0,4,7,8,11,12,14,15,16,18,19,20,21, \ldots\} ;$ all integers greater than $g(4,7)=17$ are $(4,7)$-representable.

Let $x, a, b$ be three natural numbers; we define $[x]_{a, b}$ to be the largest integer $(a, b)$-representable and no greater than $x$ :

$$
[x]_{a, b}=\max \{y \in a \mathbb{N}+b \mathbb{N}: y \leqslant x\} .
$$

By definition, an integer $x$ is $(a, b)$-representable iff $[x]_{a, b}=x$. Following the above example, we have $[10]_{4,7}=8$ and $[15]_{4,7}=15$. The set of integers $\left\{[x]_{a, b}\right\}_{0 \leqslant x \leqslant k}$ can be efficiently computed by Alg. 1 in time $\mathcal{O}(k)$. It consists in a single scan of an array of size $k$, using the property that if an integer $x$ is $(a, b)$-representable, then so are $x+a$ and $x+b$.

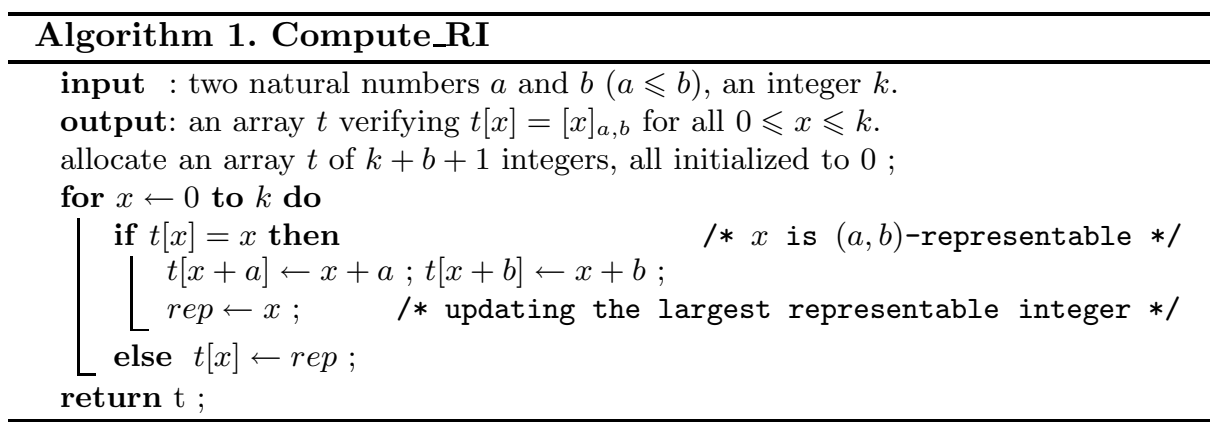

\subsection{Balls and Medial Axis}

Given a distance $d$, the ball of centre $p \in \mathbb{Z}^{n}$ and radius $r \in \mathbb{R}$ is $\mathcal{B}(p, r)=$ $\left\{q \in \mathbb{Z}^{n}: d(p, q) \leqslant r\right\}$. Since we consider discrete closed balls, any ball $B$ may have an infinite number of real radii in a left-closed interval $\left[r_{1}, r_{2}[\right.$. We define the representable radius of a given ball $B$, to be the radius of $B$ which belongs to $\operatorname{Im}(d)$, namely $r_{1}$ with the above notation. 

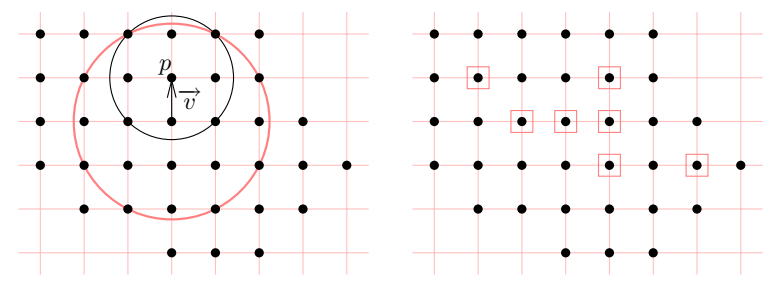

Fig. 1. A shape $\mathcal{S}$ drawn with bullets. Left: $\vec{v}$ forbids $p$ to be a MA point of $\mathcal{S}$ since $I_{p}(\mathcal{S})$ is included in $I_{p-\vec{v}}(\mathcal{S})$ (equivalently, $H_{p-\vec{v}}\left(I_{p}(\mathcal{S})\right) \subseteq \mathcal{S}$ ). Right: the medial axis of $\mathcal{S}$, i.e., the centres of maximal balls of $\mathcal{S}$. In this figure we use the Euclidean distance.

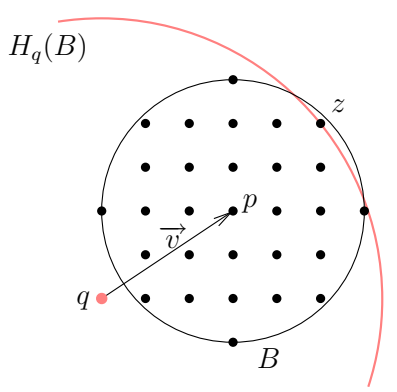

Fig. 2. $H_{q}(B)$ is the smallest ball of centre $q$ which contains $B$. The representable radius of $H_{q}(B)$ is $\mathcal{R}_{q}(B)=d(q, z)$.

Let $\mathcal{S}$ be a shape and $p \in \mathcal{S}$. We define $I_{p}(\mathcal{S})$ to be the largest ball of centre $p$ and included in $\mathcal{S}$. The inner radius of $\mathcal{S}$ is the representable radius of a largest ball included in $\mathcal{S}$. We denote by $\operatorname{CS}^{n}(R)$ the class of all $n$-dimensional shapes whose inner radius are less than or equal to $R$. A ball included in $\mathcal{S}$ is called a maximal ball of $\mathcal{S}$ if it is not included in any other ball included in $\mathcal{S}$. The Medial Axis (MA) of a shape $\mathcal{S}$ is the set of centres (and radii) of all maximal balls of $\mathcal{S}$ :

$$
p \in \operatorname{MA}(\mathcal{S}) \Leftrightarrow p \in \mathcal{S} \text { and } \forall q \in \mathcal{S} \backslash\{p\}, I_{p}(\mathcal{S}) \nsubseteq I_{q}(\mathcal{S}) .
$$

If there exists $\vec{v} \in \mathbb{Z}_{*}^{n}$ such that $I_{p}(\mathcal{S}) \subseteq I_{p-\vec{v}}(\mathcal{S})$, we say that $\vec{v}$ forbids $p$ to be a medial axis point of $\mathcal{S}$. See Fig. 1 for an example.

We denote by $H_{q}(B)$ the smallest ball of centre $q$ which contains the ball $B$; and we define $\mathcal{R}_{q}(B)$ to be the representable radius of $H_{q}(B)$, see an example in Fig. 2. Accordingly, the medial axis of $\mathcal{S}$ can be expressed as:

$$
p \in \operatorname{MA}(\mathcal{S}) \Leftrightarrow p \in \mathcal{S} \text { and } \forall q \in \mathcal{S} \backslash\{p\}, H_{q}\left(I_{p}(\mathcal{S})\right) \nsubseteq \mathcal{S} .
$$

\subsection{Chamfer Distances and Norms}

Here we recall some results from [15, Sec. 4.2 and 4.3. A chamfer mask $\mathcal{M}$ in $\mathbb{Z}^{n}$ is a central-symmetric set $\mathcal{M}=\left\{\left(\overrightarrow{v_{i}}, w_{i}\right) \in \mathbb{Z}^{n} \times \mathbb{Z}_{+*}\right\}_{1 \leqslant i \leqslant m}$ containing at least a basis of $\mathbb{Z}^{n}$, where $\left(\overrightarrow{v_{i}}, w_{i}\right)$ are called weightings, $\overrightarrow{v_{i}}$ vectors and $w_{i}$ weights. The chamfer distance $d_{\mathcal{M}}$ between two points $p, q \in \mathbb{Z}^{n}$ is

$$
d_{\mathcal{M}}(p, q)=\min \left\{\sum \lambda_{i} w_{i}: \sum \lambda_{i} \overrightarrow{v_{i}}=\overrightarrow{p q}, 1 \leqslant i \leqslant m, \lambda_{i} \in \mathbb{Z}_{+}\right\} .
$$

We write it simply $p q$ when no confusion can arise. By definition every chamfer distance is a translation invariant metric. Here we point out a link with representable integers: if $w_{1}, \ldots, w_{m}$ are the weights of a chamfer mask $\mathcal{M}$, then all representable radii belong to $w_{1} \mathbb{N}+\cdots+w_{m} \mathbb{N}$. 

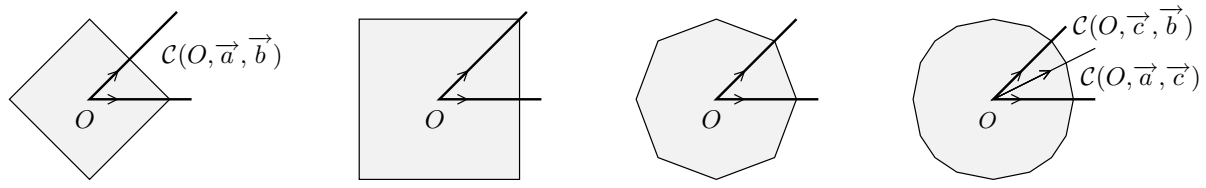

Fig. 3. Chamfer balls and influence cones for (from left to right) $d_{4}, d_{8}, d_{\langle 3,4\rangle}, d_{\langle 5,7,11\rangle}$

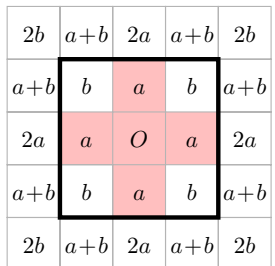

Fig. 4. Balls of radii $a$ (shaded) and $b$ (delimited by the thick line) for any minimal norm mask $\langle a, b\rangle$ (left) and $\langle a, b, c\rangle$ (right). Values indicate the chamfer distance to $O$.

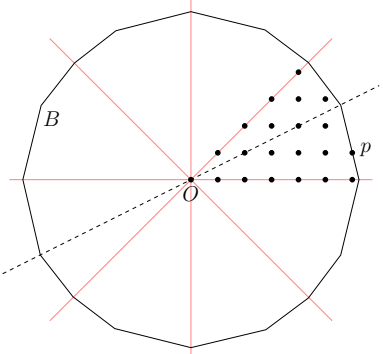

Fig. 5. A ball $B$ of radius $O p=50$ for the norm mask $\langle 8,11,18\rangle$, The generator of $B$ is drawn with bullets. 50 is $(8,18)$ representable: $50=4 * 8+18$. The dashed line represents the axis $O+\mathbb{R} \vec{c}$.

Consider $\mathcal{M}^{\prime}=\left\{O+\overrightarrow{v_{i}} / w_{i}\right\}_{1 \leqslant i \leqslant m} \in \mathbb{R}^{n}$ and let $B_{\mathcal{M}}^{\prime}=\operatorname{conv}\left(\mathcal{M}^{\prime}\right)$, then $B_{\mathcal{M}}^{\prime}$ is a central-symmetric and convex polyhedron whose facets separate $\mathbb{R}^{n}$ in cones from $O$. A facet $\mathcal{F}$ of $B_{\mathcal{M}}^{\prime}$ is generated by a subset $\left.\mathcal{M}\right|_{\mathcal{F}}=\left\{\left(\overrightarrow{v_{j}}, w_{j}\right)\right\}_{1 \leqslant j \leqslant n}$ of $\mathcal{M}$; if $\Delta_{\mathcal{F}}=\operatorname{det}\left\{\overrightarrow{v_{j}}\right\}_{1 \leqslant j \leqslant n}$ is such that $\left|\Delta_{\mathcal{F}}\right|=1$, then $\mathcal{F}$ is said unimodular. If each facet of $B_{\mathcal{M}}^{\prime}$ is unimodular, then $d_{\mathcal{M}}$ is a norm in $\mathbb{Z}^{n}$ ([15], p. 53). We denote by $\|\vec{v}\|$ the chamfer norm of a vector $\vec{v}$.

Now let $d_{\mathcal{M}}$ be a chamfer norm, $\mathcal{F}$ a facet of $B_{\mathcal{M}}^{\prime}$ and $\left.\mathcal{M}\right|_{\mathcal{F}}=\left\{\left(\overrightarrow{v_{j}}, w_{j}\right)\right\}_{1 \leqslant j \leqslant n}$; then for each point $p=\left(p_{1}, \ldots, p_{n}\right)$ in the cone $(O, \mathcal{F})$, called influence cone of $\left.\mathcal{M}\right|_{\mathcal{F}}$, we have $d_{\mathcal{M}}(O, p)=p_{1} \delta_{1}+\cdots+p_{n} \delta_{n}$, where $\left(\delta_{1}, \ldots, \delta_{n}\right)=\overrightarrow{\delta_{\mathcal{F}}}$ is a normal vector of $\mathcal{F}$, and $\delta_{k}$ is the elementary displacement for the $k^{\text {th }}$ coordinate:

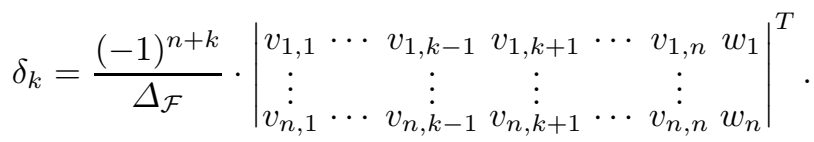

In other words, if a vector $\vec{v}$ belongs to some influence cone $\mathcal{C}\left(\overrightarrow{v_{1}}, \ldots, \overrightarrow{v_{n}}\right)$, then there is a minimal path from $O$ to $O+\vec{v}$ which is only composed of the vectors $\overrightarrow{v_{1}}, \ldots, \overrightarrow{v_{n}}$, and so $\|\vec{v}\|$ is $\left(w_{1}, \ldots, w_{n}\right)$-representable.

From now on, we restrict the discussion to G-symmetrical masks. As a consequence, the chamfer balls are G-symmetrical. In $\mathbb{Z}^{2}$, let $\vec{a}=(1,0), \vec{b}=(1,1)$, $\vec{c}=(2,1)$; a common way to denote $3 \times 3$ masks is $\langle a, b\rangle=\{(\vec{a}, a),(\vec{b}, b)\}$ (plus the G-symmetrical weightings) and $\langle a, b, c\rangle=\{(\vec{a}, a),(\vec{b}, b),(\vec{c}, c)\}$ 
(ditto) for $5 \times 5$ masks. Note that $\mathrm{G}\left(\mathbb{Z}^{2}\right)=\mathcal{C}(\vec{a}, \vec{b})$. Widely used chamfer norms are $d_{4}=\ell_{1}=d_{\langle 1,2\rangle}, d_{8}=\ell_{\infty}=d_{\langle 1,1\rangle}, d_{\langle 3,4\rangle}$ and $d_{\langle 5,7,11\rangle}$, see Fig. 3. Chamfer distances to $O$ are indicated in Fig. 4 for $\langle a, b\rangle$ and $\langle a, b, c\rangle$ norm masks. Also, Fig. 5 illustrates a ball of radius 50 for the norm mask $\langle 8,11,18\rangle$. The conditions for a chamfer mask $\langle a, b\rangle$ to generate a norm are $a \leqslant b \leqslant 2 a$; and according to (5), the elementary displacements in $\mathrm{G}\left(\mathbb{Z}^{2}\right)$ are $\delta_{x}=a$ and $\delta_{y}=b-a$. For $\langle a, b, c\rangle$ norm masks the constraints are $\{2 a \leqslant c ; c \leqslant a+b ; 3 b \leqslant 2 c\}$, while the displacements are $\left\{\delta_{x}=a, \delta_{y}=c-2 a\right\}$ in the influence cone $\mathcal{C}(\vec{a}, \vec{c})$ and $\left\{\delta_{x}=c-b, \delta_{y}=2 b-c\right\}$ in the influence cone $\mathcal{C}(\vec{c}, \vec{b})$.

Without loss of generality, we will only consider minimal chamfer masks. A mask $\mathcal{M}=\left\{\left(\overrightarrow{v_{i}}, w_{i}\right)\right\}_{1 \leqslant i \leqslant m}$ is said minimal if the removal of any single weighting of $\mathcal{M}$ modifies the distance, i.e, if $\forall 1 \leqslant i \leqslant m, d_{\mathcal{M}} \neq d_{\mathcal{M} \backslash\left\{\left(\overrightarrow{v_{i}}, w_{i}\right)\right\}}$. Considering Fig. 4, it is easily seen that a norm mask $\langle a, b\rangle$ is minimal iff $b<2 a$. Otherwise we would have $b=2 a$, so $(1,0)+(0,1)$ would be a minimal chamfer path from $O$ to $(1,1)$, hence $\vec{b}$ would be redundant in the chamfer mask. In a similar way, we check at once that a norm mask $\langle a, b, c\rangle$ is minimal iff $c<a+b$.

Here are some elementary properties of covering relations between chamfer balls, illustrated in Fig. 6. These properties are extensively used in the proofs.

Lemma 1 (Representable radius). Let $\mathcal{C}\left(\overrightarrow{v_{1}}, \overrightarrow{v_{2}}\right)$ be an influence cone of a given 2D chamfer norm, and assume the vectors $\overrightarrow{v_{1}}$ and $\overrightarrow{v_{2}}$ have respective weights $w_{1}$ and $w_{2}$. If $r$ is $\left(w_{1}, w_{2}\right)$-representable then for any vector $\vec{v}$ in $\mathcal{C}\left(\overrightarrow{v_{1}}, \overrightarrow{v_{2}}\right)$, we have $\mathcal{R}_{O-\vec{v}}(\mathcal{B}(O, r))=r+\|\vec{v}\|$.

Lemma 2 (Covering a cone). Let $\mathcal{C}\left(\overrightarrow{v_{1}}, \overrightarrow{v_{2}}\right)$ be an influence cone of a given $2 D$ chamfer norm, and $B$ be a ball of centre $O$. For any vector $\vec{v}$ in the cone $\mathcal{C}\left(\overrightarrow{v_{1}}, \overrightarrow{v_{2}}\right)$, we have $B \cap \mathcal{C}\left(O, \overrightarrow{v_{1}}, \overrightarrow{v_{2}}\right)=H_{O-\vec{v}}(B) \cap \mathcal{C}\left(O, \overrightarrow{v_{1}}, \overrightarrow{v_{2}}\right)$.

In other words, the balls $B$ and $H_{O-\vec{v}}(B)$ coincide in the cone $\mathcal{C}\left(O, \overrightarrow{v_{1}}, \overrightarrow{v_{2}}\right)$.
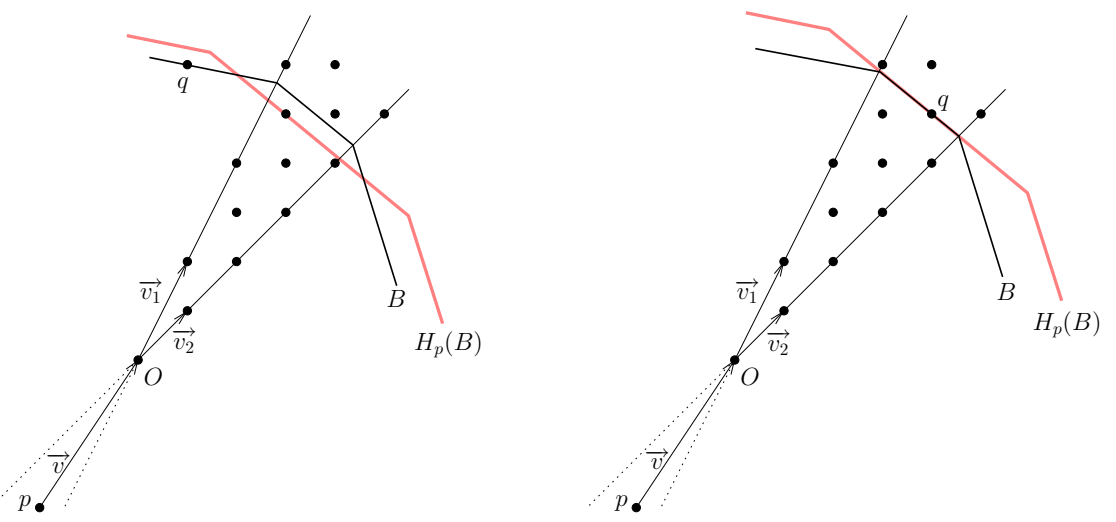

Fig. 6. Covering a ball $B$ of center $O$ and representable radius $r=d(O, q)$ in direction $\vec{v}$, with $\vec{v}$ being a vector in the influence cone $\mathcal{C}\left(\overrightarrow{v_{1}}, \overrightarrow{v_{2}}\right)$. Left: $r$ is not $\left(w_{1}, w_{2}\right)$ representable so $\mathcal{R}_{p}(B)<r+\|\vec{v}\|$. Right: $r$ is $\left(w_{1}, w_{2}\right)$-representable, so there is a minimal chamfer path from $p$ to $q$ passing through $O$, hence $\mathcal{R}_{p}(B)=r+\|\vec{v}\|$. 


\section{The Medial Axis Test Mask $\mathcal{T}_{\mathcal{M}}$}

Given a chamfer mask $\mathcal{M}$ in $\mathbb{Z}^{n}$, we define the test mask $\mathcal{T}_{\mathcal{M}}(R)$ to be the minimum neighbourhood sufficient to detect locally, for any $\mathcal{S}$ in $\operatorname{CS}^{n}(R)$ and any $p \in \mathcal{S}$, if $p$ is a point of $\operatorname{MA}(\mathcal{S})$ :

$$
\left\{\begin{array}{l}
\forall \mathcal{S} \in \mathrm{CS}^{n}(R), \forall p \in \mathcal{S},\left(p \notin \mathrm{MA}(\mathcal{S}) \Rightarrow \exists \vec{v} \in \mathcal{T}_{\mathcal{M}}(R), I_{p}(\mathcal{S}) \subseteq I_{p-\vec{v}}(\mathcal{S})\right) \\
\mathcal{T}_{\mathcal{M}}(R) \text { has minimum cardinality. }
\end{array}\right.
$$

If $p \in \operatorname{MA}(\mathcal{S})$, then no $\vec{v} \in \mathbb{Z}^{n}$ satisfies $I_{p}(\mathcal{S}) \subseteq I_{p-\vec{v}}(\mathcal{S})$. Otherwise, if $p \notin$ $\operatorname{MA}(\mathcal{S})$, then $\mathcal{T}_{\mathcal{M}}(R)$ contains at least one vector $\vec{v}$ which forbids $p$ from $\operatorname{MA}(\mathcal{S})$. We have shown the unicity of $\mathcal{T}_{\mathcal{M}}(R)$ for all $R \geqslant 0$ in 14] (the proof remains the same for any norm). As a corollary, if the considered norm is G-symmetrical, then so is $\mathcal{T}_{\mathcal{M}}(R)$.

Finally, we set $\mathcal{T}_{\mathcal{M}}=\lim _{R \rightarrow+\infty} \mathcal{T}_{\mathcal{M}}(R)$. We write it simply $\mathcal{T}$ when no confusion can arise, and by abuse of notation we often write $\mathcal{T}$ instead of $\mathrm{G}(\mathcal{T})$. The appearance radius $R_{a p p}(\vec{v})$ of a given vector $\vec{v}$ is the smallest radius $R$ for which $\vec{v} \in \mathcal{T}_{\mathcal{M}}(R)$.

Definition 1 (Domination). We write $B_{r}$ for $\mathcal{B}(O, r)$. Given a distance $d$, a vector $\vec{v} \in \mathbb{Z}_{*}^{n}$ is dominated by a vector $\vec{u} \in \mathbb{Z}_{*}^{n}$ if for all radii $r \in \mathbb{R}_{+}$, $H_{O-\vec{u}}\left(B_{r}\right)$ is strictly included in $H_{O-\vec{v}}\left(B_{r}\right)$ :

$$
\vec{v} \prec \vec{u} \Leftrightarrow \forall r \geqslant 0, H_{O-\vec{u}}\left(B_{r}\right) \subsetneq H_{O-\vec{v}}\left(B_{r}\right) .
$$

Note that by definition, $\prec$ is transitive. The basic idea of the domination relation is the following: if $\vec{v} \prec \vec{u}$, it is useless to test if a given point $p$ belongs to some $\operatorname{MA}(\mathcal{S})$ in both directions $\vec{u}$ and $\vec{v}$, testing in direction $\vec{u}$ is sufficient. As a consequence, $\vec{v} \prec \vec{u}$ implies that $\vec{v}$ does not belong to $\mathcal{T}$.

In the remainder of the paper, we will restrict the discussion to the discrete plane $\mathbb{Z}^{2}$. First, we examine the simple case of $3 \times 3$ chamfer norms. We know from [1] that for $d_{4}=d_{\langle 1\rangle}$ and $d_{8}=d_{\langle 1,1\rangle}$, the test masks $\mathcal{T}$ are respectively $\{\vec{a}\}$ and $\{\vec{a}, \vec{b}\}$. In the remainder of the section we examine the general $\langle a, b\rangle$ minimal chamfer masks, i.e., which satisfy $a<b<2 a$.

The simplicity of the $3 \times 3$ masks comes from the fact that there is only one influence cone $\mathcal{C}(\vec{a}, \vec{b})$ in the generator. As a consequence, Lemma 1 always applies (see Fig. 17), and we obtain the domination relation:

Lemma 3. Let $\mathcal{M}$ be a minimal norm mask $\langle a, b\rangle$, then we have: $\forall \vec{u}, \vec{v} \in$ $\mathrm{G}\left(\mathbb{Z}_{*}^{2}\right), \vec{u} \succ \vec{u}+\vec{v}$.

For these norms, it is also easy to check that $\vec{a}$ and $\vec{b}$ appear in $\mathcal{T}$ for respective radius $a$ and $b$ : the fact that $a<b$ implies that the balls $B_{a}$ of radii $a$ and centre

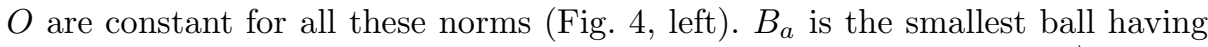
positive radius, and $p(1,0)$ does not belong to $\operatorname{MA}\left(B_{a}\right)$ so $\mathcal{T}(a)=\{\vec{a}\}$. Same reasonning applies to $B_{b}=\mathcal{B}(O, b)$ : these balls are constant for all these norms 


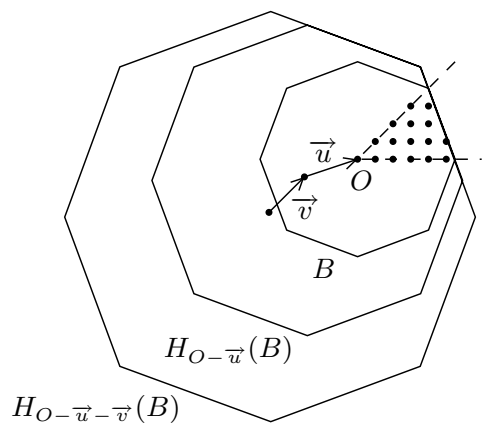

Fig. 7. For any $\langle a, b\rangle$ norm mask and $\vec{u}, \vec{v} \in \mathrm{G}\left(\mathbb{Z}^{2}\right), \vec{u}$ dominates $\vec{u}+\vec{v}$

$(b<2 a)$. Since $\vec{b}$ is the only vector which forbids $q(1,1)$ from $\operatorname{MA}\left(B_{b}\right)$, we conclude that $\mathcal{T}(b)=\{\vec{a}, \vec{b}\}$.

Lemma 3 asserts that any vector $\vec{v} \in \mathrm{G}\left(\mathbb{Z}_{*}^{2}\right)$ is dominated by $\vec{a}$ or by $\vec{b}$ (or both). Adding the fact that $\mathcal{T}(b)=\{\vec{a}, \vec{b}\}$, it follows:

Theorem 1. For any minimal norm mask $\mathcal{M}=\langle a, b\rangle$, we have $\mathcal{T}_{\mathcal{M}}=\{\vec{a}, \vec{b}\}$.

\section{Test Masks for $5 \times 5$ Chamfer Norms}

\subsection{The Test Masks $\mathcal{T}_{\langle a, b, c\rangle}$ Are Bounded}

Compared to the case of $3 \times 3$ minimal norm masks, same reasonning holds here concerning the appearance of $\vec{a}$ and $\vec{b}$. Since $b \leqslant \frac{2}{3} c<c$ for $\langle a, b, c\rangle$ norms, balls of radii $a$ and $b$ are the same as for $\langle a, b\rangle$ masks (see Fig. 4 right). Thus, we have $\mathcal{T}(b)=\{\vec{a}, \vec{b}\}$. On the other hand, the domination relations are described in the following two lemmas:

Lemma 4 (Domination along $\vec{a} \mathbb{N}$ and $\vec{b} \mathbb{N}$ ). For any minimal norm mask $\langle a, b, c\rangle$ and any $k \in \mathbb{N}_{*}$, we have $k \vec{a} \succ(k+1) \vec{a}$ and $k \vec{b} \succ(k+1) \vec{b}$.

Lemma 5 (Domination by addition of $\vec{c}$ ). For any minimal norm mask $\langle a, b, c\rangle$ and any $\vec{v} \in \mathrm{G}\left(\mathbb{Z}_{*}^{2}\right)$, we have $\vec{v} \succ \vec{v}+\vec{c}$.

Let us mention that in the Euclidean case, the Lemma 4 holds for any vector 14. However, this is generally false for chamfer norms. Geometrically speaking, this lemma holds for $\vec{a}$ and $\vec{b}$ because we consider G-symmetrical masks and each of these two vectors has the same direction as a G-symmetry axis of $\mathbb{Z}^{2}$.

Lemma 5 comes from the following observation: since the generator only contains two influence cones $\mathcal{C}(\vec{a}, \vec{c})$ and $\mathcal{C}(\vec{c}, \vec{b})$, the vector $\vec{c}$ belongs to all influence cones of $\mathrm{G}\left(\mathbb{Z}^{2}\right)$. Accordingly, for any point $p \in \mathrm{G}\left(\mathbb{Z}^{2}\right)$ which does not 
lie on the $O+\vec{a} \mathbb{N}$ nor the $O+\vec{b} \mathbb{N}$ axis, any minimal chamfer path from $O$ to $p$ contains at least one occurrence of $\vec{c}$.

According to these domination relations, and the transitivity of $\prec$, we obtain:

Theorem 2. For any minimal norm mask $\mathcal{M}=\langle a, b, c\rangle$, we have

$$
\{\vec{a}, \vec{b}\} \subseteq \mathcal{T}_{\mathcal{M}} \subseteq\{\vec{a}, \vec{b}, \vec{c}\}
$$

We observe that concerning test masks, the only difference between $3 \times 3$ and $5 \times 5$ chamfer norms is the possible appearance of $\vec{c}$ in the case of $5 \times 5$ norms. The following section establishes a relation between the appearance of $\vec{c}$ and the notion of representable integers.

\subsection{Appearance of $\vec{c}$ in the Test Masks $\mathcal{T}_{\langle a, b, c\rangle}$}

The task is now to find all the chamfer masks $\mathcal{M}=\langle a, b, c\rangle$ for which $\vec{c} \in \mathcal{T}_{\mathcal{M}}$. We first illustrate the appearance of $\vec{c}$ for $\langle 5,7,11\rangle$ in Fig. 8 . $B$ is the ball of centre $O$ and radius 35 . On the left is shown $H_{O-\rightarrow}(B)$ : there is a point of $B$ in the cone $\mathcal{C}(O, \vec{a}, \vec{c})$ which is at distance 35 from $O(35$ is $(5,11)$-representable). Hence Lemma 1 yields $\mathcal{R}_{O-\rightarrow}(B)=35+5=40$. However, there is a point $p$ in the cone $\mathcal{C}(O, \vec{c}, \vec{b})$ at distance 40 from $O$, so $q=p-\vec{a}$ (circled point) belongs to $H_{O-\rightarrow}(B)$. On the other hand, $q$ is at a distance 36 from $O$, so $q \notin B$. Given that $B$ and $H_{O-} \vec{c}(B)$ are equal in $\mathcal{C}(O, \vec{c}, \vec{b})$ (using $\vec{v}=\vec{c}$ in Lemma 2), the point $q$ does not belong to $H_{O-\vec{c}}(B)$.

The same conclusion can be drawn with $H_{O-\vec{b}}(B)$ on the right of the figure: the circled point $q$ belongs to $H_{O-\vec{b}}(B)$, but not to $B$.

The configuration of Fig 8 is the smallest one for which neither $H_{O-\vec{a}}(B)$ nor $H_{O-\vec{b}}(B)$ are included in $H_{O-\vec{c}}(B)$, implying that the appearance radius of $\vec{c}$ is $\mathcal{R}_{O-\vec{c}}(B)=35+c=46$. This phenomenon can be expressed in an arithmetical way:

Lemma 6 (Arithmetical expression of $R_{a p p}(\vec{c})$ ). For any minimal norm mask $\langle a, b, c\rangle$, we have

$$
R_{a p p}(\vec{c})=\min \left\{r \in \mathbb{N}:\left\{\begin{array}{l}
r+c-b<\left[[r]_{a, c}+a\right]_{b, c} \\
r+c-a<\left[[r]_{b, c}+b\right]_{a, c}
\end{array}\right\}+c .\right.
$$

This lemma is the starting point of all remaining results of this paper. In the next section, this lemma will be used to deduce an upper bound of the appearance radius of $\vec{c}$, as well as a simple algorithm for computing $R_{a p p}(\vec{c})$. First, we provide a criterion to determine whether the system in (8) has a solution:

Theorem 3 (Appearance of $\vec{c}$ ). For any minimal norm mask $\mathcal{M}=\langle a, b, c\rangle$,

$$
\vec{c} \in \mathcal{T}_{\mathcal{M}} \Leftrightarrow \operatorname{gcd}(a, c)+\operatorname{gcd}(b, c) \leqslant 2(a+b-c) .
$$



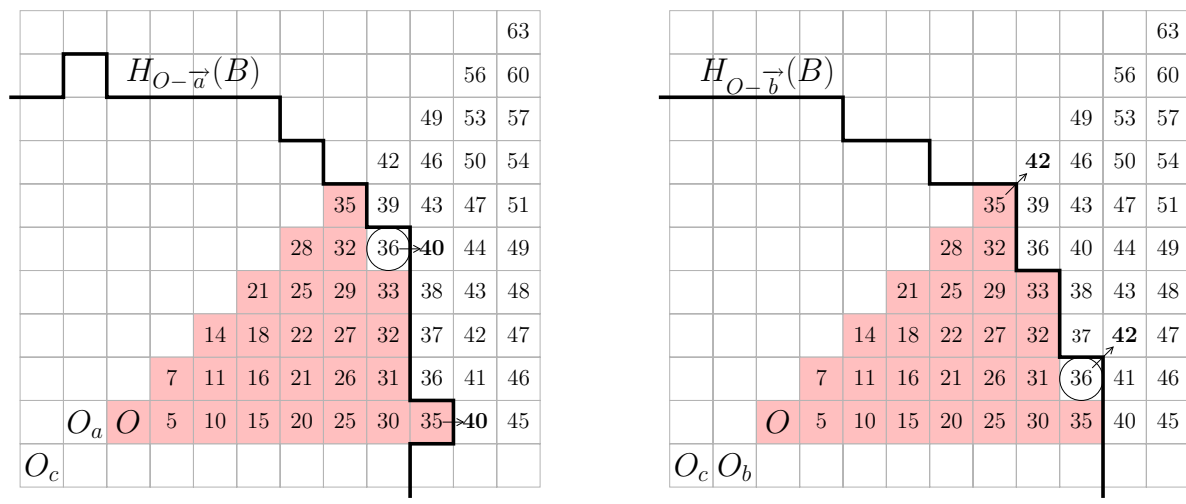

Fig. 8. Appearance of $\vec{c}$ for the mask $\langle 5,7,11\rangle$. A ball $B$ of centre $O$ and radius 35 (whose generator is shaded), the points $O_{a}=O-\vec{a}, O_{b}=O-\vec{b}$ and $O_{c}=O-\vec{c}$. Left : the circled point belongs to $H_{O-\vec{a}}(B)$ but not to $B$. Right : the circled point belongs to $H_{O-\vec{b}}(B)$ but not to $B$.

\section{Appearance Radius of $\vec{c}$}

\subsection{A Bound for the Appearance Radius of $\vec{c}$}

We now combine Lemma 6 and Sylvester's result on the Frobenius problem to obtain an upper bound of $R_{a p p}(\vec{c})$.

In the case were $\operatorname{gcd}(a, c)=\operatorname{gcd}(b, c)=1$, it is easy to check that the system in (8) is satisfied for $r=g(b, c)+1$, since for all $r>g(b, c),\left[[r]_{a, c}+a\right]_{b, c}=$ $[r+a]_{b, c}=r+a$, and similarly $\left[[r]_{b, c}+b\right]_{a, c}=r+b$. In this case, the system in (8) is therefore equivalent to $c<a+b$, which is a necessary condition for the minimality of $\langle a, b, c\rangle$. It follows from Lemma 6 that $R_{a p p}(\vec{c}) \leqslant g(b, c)+1+c$. Then, applying Sylvester's result $g(b, c)=b c-b-c$ gives $R_{a p p}(\vec{c})<b c$.

We can generalize this property to the remaining masks:

Theorem 4. Let $\mathcal{M}=\langle a, b, c\rangle$ be a minimal norm mask. If $\vec{c} \in \mathcal{T}_{\mathcal{M}}$ then $R_{a p p}(\vec{c})<b c$.

\subsection{Computation of $R_{a p p}(\vec{c})$}

Algorithm 2 computes the appearance radius of $\vec{c}$ in the MA test mask for any minimal norm mask $\mathcal{M}=\langle a, b, c\rangle$. The presence of $\vec{c}$ in the test mask (line 1) is determined according to Thm. 3. The calls Compute_RI (a,c, max) and Compute_RI $(b, c, \max )$ are computed in time $\mathcal{O}(\max )=\mathcal{O}(b c)$, see Alg. 1 The main loop (lines 5-7) makes the arithmetical test provided by Lemma 6 , for each radius $r$, from $r=0$ to $r=R_{a p p}(\vec{c})-c<b c-c$ (the bound comes from Thm. 44). Therefore, the time complexity of Alg. 2 is $\mathcal{O}(b c)$. As an example, this algorithm is run for $\langle 5,7,11\rangle$ in Fig. 9. 

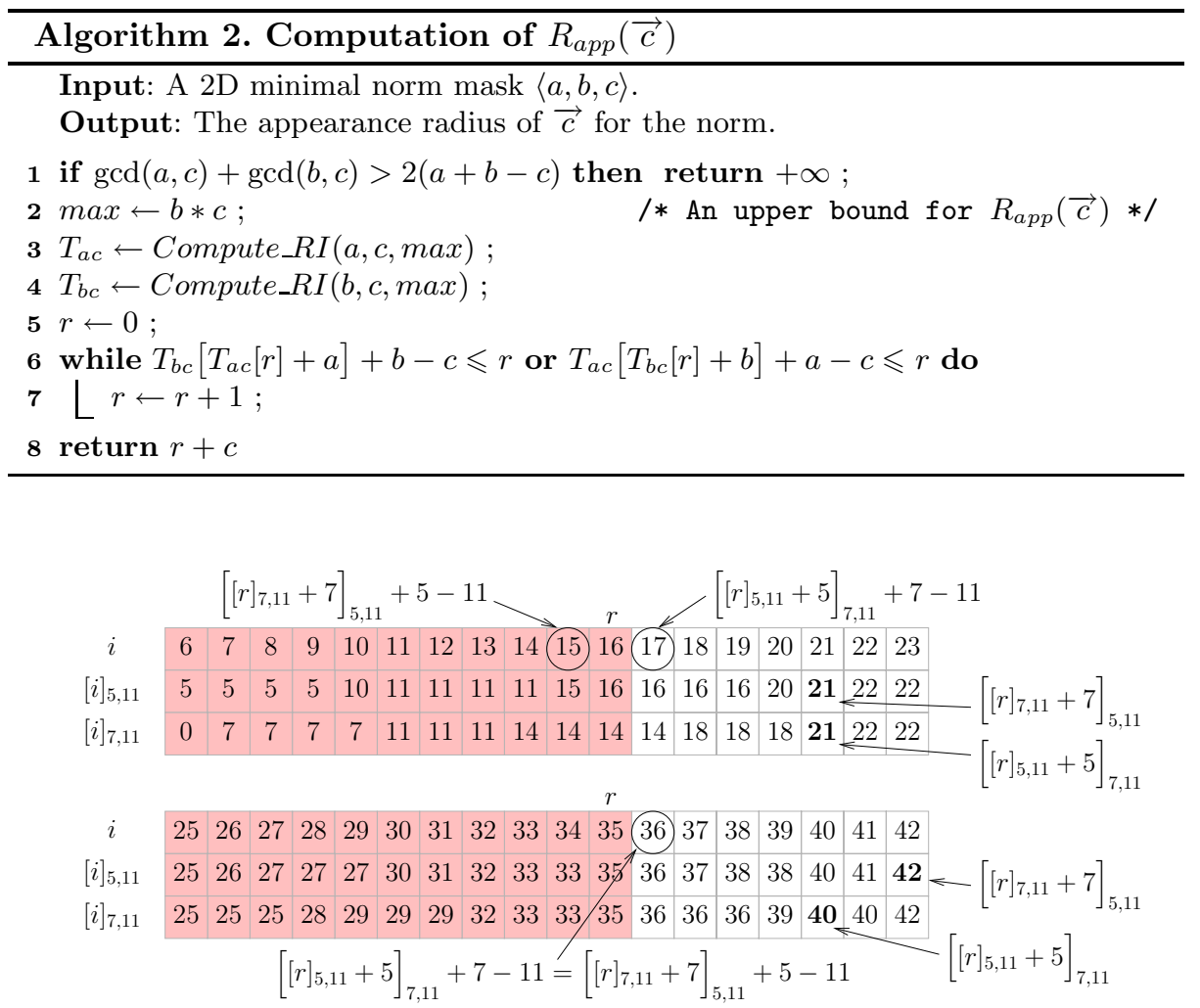

Fig. 9. Execution of Alg. 2 for the mask $\langle 5,7,11\rangle$. The arrays $T_{a c}[$.$] et T_{b c}[$.$] respectively$ contain the values of $[.]_{5,11}$ and $[.]_{7,11}$. Top: when $r=16$ (configuration illustrated in Fig. 10). Bottom: when it detects the configuration of Fig. 8 (using same graphical rules), that is to say, for the appearance of $\vec{c}$ in $\mathcal{T}_{\langle 5,7,11\rangle}$.
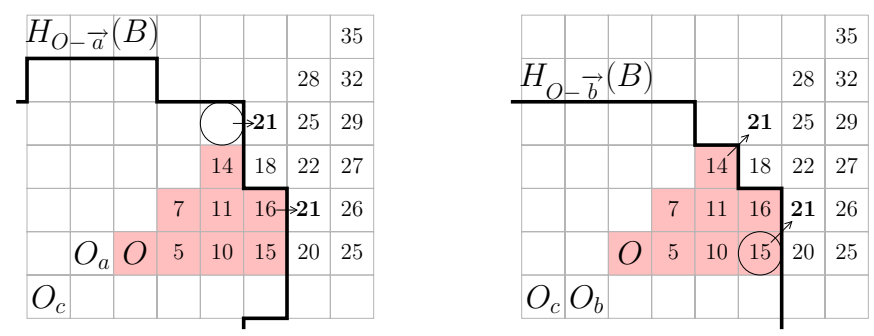

Fig. 10. Configuration for $\langle 5,7,11\rangle$ and $r=16$. $B$ is the ball of centre $O$ and radius $r$, its generator is shaded. Left: the circled point shows the result of $x=\left[[r]_{a, c}+a\right]_{b, c}+$ $b-c=17$. Since $x>r$, this point belongs to $H_{O-\vec{a}}(B) \backslash H_{O-\vec{c}}(B)$. Right: the circled point shows the result of $x=\left[[r]_{b, c}+b\right]_{a, c}+a-c=15$. Since $x \leqslant r$, there is no point in $H_{O-\vec{b}}(B) \backslash H_{O-\vec{c}}(B)$. So this configuration does not lead to the appearance of $\vec{c}$. 


\section{Conclusion}

We have characterized the MA test masks of all 2D chamfer norms $\langle a, b\rangle$ and $\langle a, b, c\rangle$. The next step is devoted to larger 2D masks. We have noticed that simple $7 \times 7$ chamfer masks (created by adding a single weighting $((3,1), d)$ or $((3,2), e)$ to a $5 \times 5$ mask) generally yield test masks with new properties: they can be larger than the chamfer mask itself, and can also contain non-visible vectors. We intend to use our arithmetical framework to describe these test masks.

\section{References}

1. Pfaltz, J.L., Rosenfeld, A.: Computer Representation of Planar Regions by their Skeletons. Comm. of ACM 10, 119-125 (1967)

2. Coeurjolly, D., Hulin, J., Sivignon, I.: Finding a Minimum Medial Axis of a Discrete Shape is NP-hard. Theoretical Computer Science 206(1-2), 72-79 (2008)

3. Coeurjolly, D., Montanvert, A.: Optimable Separable Algorithms to compute the Reverse Euclidean Distance Transformation and Discrete Medial Axis in Arbitrary Dimension. IEEE Trans. on PAMI 29(3), 437-448 (2007)

4. Saúde, A.V., Couprie, M., Lotufo, R.A.: Discrete 2D and 3D euclidean medial axis in higher resolution. Image Vision Comput. 27(4), 354-363 (2009)

5. Borgefors, G.: Distance Transformations in Arbitrary Dimensions. Computer Vision, Graphics and Image Processing 27, 321-345 (1984)

6. Hirata, T.: A Unified Linear-time Algorithm for Computing Distance Maps. Information Processing letters 58(3), 12-133 (1996)

7. Arcelli, C., Sanniti di Baja, G.: Finding Local Maxima in a Pseudo-Euclidean Distance Transform. Comp. Vision, Graphics and Image Proc. 43, 361-367 (1988)

8. Borgefors, G.: Centres of Maximal Disks in the 5-7-11 Distance Transform. In: $8^{\text {th }}$ Scandinavian Conf. on Image Analysis, Troms $\varnothing$, Norway, pp. 105-111 (1993)

9. Rémy, E., Thiel, E.: Medial Axis for Chamfer Distances: Computing Look-up Tables and Neighbourhoods in 2D or 3D. Pattern Rec. Letters 23(6), 649-661 (2002)

10. Rémy, E., Thiel, E.: Exact Medial Axis with Euclidean Distance. Image and Vision Computing 23(2), 167-175 (2005)

11. The Npic library and tools, http://www.lif.univ-mrs.fr/ thiel/npic/

12. Normand, N., Évenou, P.: Medial Axis LUT Computation for Chamfer Norms using H-polytopes. In: Coeurjolly, D., Sivignon, I., Tougne, L., Dupont, F. (eds.) DGCI 2008. LNCS, vol. 4992, pp. 189-200. Springer, Heidelberg (2008)

13. Normand, N., Évenou, P.: Medial Axis Lookup Table and Test Neighborhood Computation for 3D Chamfer Norms. Pattern Rec. 42(10), 2288-2296 (2009)

14. Hulin, J., Thiel, E.: Visible Vectors and Discrete Euclidean Medial Axis. Discrete and Computational Geometry (in press) (Available online December 10, 2008)

15. Thiel, E.: Géométrie des Distances de Chanfrein. Habilitation à Diriger les Rech., Univ. de la Méditerranée, (déc 2001), http://www.lif.univ-mrs.fr/ thiel/hdr/

16. Ramírez Alfonsín, J.L.: The Diophantine Frobenius Problem. Oxford Lectures Series in Mathematics and its Applications 30 (2005)

17. Annex with proofs: http://pageperso.lif.univ-mrs.fr/ jerome.hulin/dgci09/ 